\title{
Development of spontaneous magnetism and half-metallicity in monolayer $\mathrm{MoS}_{2}$
}

\author{
Altaf Ur Rahman and Gul Rahman* \\ Department of Physics, Quaid-i-Azam University, Islamabad 45320, Pakistan \\ Víctor M. García-Suárez \\ Departamento de Física, Universidad de Oviedo, 33007 Oviedo Spain and \\ Nanomaterials and Nanotechnology Research Center (CINN), Spain
}

\begin{abstract}
Half-metallic behavior and ferromagnetism are predicted in strained $\mathrm{MoS}_{2}$ with different light elements adsorbed using density functional theory. We find that strain increases the density of states at the Fermi energy for $Y$ doping $(Y=\mathrm{H}, \mathrm{Li}$, and $\mathrm{F})$ at the $\mathrm{S}$ sites and strain-driven magnetism develops in agreement with the Stoner mean field model. Strain-driven magnetism requires less strain $(\sim 3 \%)$ for $\mathrm{H}$ doping as compared with $\mathrm{F}$ and $\mathrm{Li}$ doping. No saturation of the spin-magnetic moment is observed in Li-doped $\mathrm{MoS}_{2}$ due to less charge transfer from the Mo $d$ electrons and the added atoms do not significantly increase the Spin-orbit coupling. Half-metallic ferromagnetism is predicted in $\mathrm{H}$ and $\mathrm{F}$-doped $\mathrm{MoS}_{2}$. Fixed magnetic moments calculations are also performed, and the DFT computed data is fitted with the Landau mean field theory to investigate the emergence of spontaneous magnetism in $Y$-doped $\mathrm{MoS}_{2}$. We predict spontaneous magnetism in systems with large (small) mag netic moments for $\mathrm{H} / \mathrm{F}(\mathrm{Li})$ atoms. The large (small) magnetic moments are atttributed to the electronegativity difference between $\mathrm{S}$ and $Y$ atoms. These results suggest that $\mathrm{H}$ and $\mathrm{F}$ adsorbed monolayer $\mathrm{MoS}_{2}$ is a good candidate for spin-based electronic devices.
\end{abstract}

*Eulrahman@qau.edu.pk 


\section{INTRODUCTION}

Magnetism remains as one of the areas of condensed matter physics that has been a hot issue since its discovery. Much efforts have been made to understand its origin in different materials, such as those with conventional $d$ or $f$ electrons. However, magnetism induced by $p$ electrons (i.e., light elements, C, N...etc) in nonmagnetic hosts is still under progress. The main advantage of $p$ electron magnetism is the low formation energy [1] and the absence of magnetic clusters, which usually occur in $d$ electron magnetic materials [2]. Inducing magnetism either through doping or vacancies is also more favourable in nano-structured materials than in bulk systems [3]. The discovery of two-dimensional (2D) materials such as graphene, has triggered an extensive study of magnetism on 2D nanoscale materials for applications in next-generation nano devices because of their easy fabrication [4].

Much work has been devoted to explore graphene in electronic devices, however, due to the absence of a bandgap the interests of researchers has shifted to explore new 2D materials [5] such as silicene. Modern optoelectronic devices require however materials with a direct bandgap that can be tuned by applying an electric field [6]. Among the newly discovered 2D materials, 2D transition metal dichalcogenides (TMDs) have attracted great attention because of their layered crystal structure, which allows the tuning of their electronic properties by either covalent doping [7], adsorption between the weakly bonded layers [8], or fabrication of thin nanostructured materials [5]. TMDs, which have a molecular formula $\mathrm{TM}-\mathrm{X}_{2}$ (TM is the set of transition metals Mo, W, Ti, Ta, Zr, Nb, Re, Ni, etc, and X is the set of chalcogen atoms $\mathrm{O}, \mathrm{S}, \mathrm{Se}, \mathrm{Te})$ [4-8, 13-17], are a new family of 2D materials which have a graphene-like 2D structure. Single layers are $3 \AA$ thick, whereas the interlayer distance is about $6.5 \AA$ [18]. Within a monolayer, the Mo and S atoms make a 2D hexagonal sublattice. From such structures, the 3D material is formed by vertically stacking 2D layers through weak van der Waals interactions. Different crystal structures are possible depending on the stacking sequence of atoms along the $c$ axis. The most common structures occur in trigonal prismatic $(2 \mathrm{H})$ and rhombohedral $(3 \mathrm{R})$ phases, being the former (2H trigonal prismatic) the most stable [19].

Monolayer $\mathrm{MoS}_{2}$ triggered a lot of attention in recent years due to its applications in the field of optoelectronics [13], valleytronics [14], and spintronics [15, 16]. MoS $\mathrm{M}_{2}$ FETs with high current ON/OFF ratios, high-sensitivity phototransistors, logic circuits, and amplifiers 
based on monolayer- $\mathrm{MoS}_{2}$ have also been recently demonstrated [20]. Previous studies show that bulk $\mathrm{MoS}_{2}$ is an indirect bandgap $(1.2 \mathrm{eV})$ semiconductor [7], while $2 \mathrm{D} \mathrm{MoS}_{2}$ is a direct bandgap semiconductor $(1.8 \mathrm{eV})[4-8,13-22]$. This last one can be therefore regarded as an ideal candidate for nano electronic devices. The band gap transition (indirect to direct) of bulk $\mathrm{MoS}_{2}$ can be tuned by changing the number of layers [23]. In contrast to graphene, $\mathrm{MoS}_{2}$ has therefore a tunable band gap that can also be changed by altering the composition [24], functionalization [25] or applying external fields [26]. For electronic devices, a monolayer of $\mathrm{MoS}_{2}$ requires $n$-type or $p$-type doping [27]. Additionally, for spintronic devices $\mathrm{MoS}_{2}$ requires doping/adsorption and strain engineering to achieve a magnetic state [8]. Functionalization of $2 \mathrm{D}$ materials through adsorption of light elements have interesting properties, ranging from metallicity to superconductivity [9-12]. Recent theoretical calculations show that $\mathrm{Li}$ can form clusters when adsorbed at graphene substrate[9], and such clustering can be degraded either by the application of external electric field or doping[9]. On the other hand $\mathrm{Na}, \mathrm{K}, \mathrm{Ca}$ in graphene show uniform distribution as well as quasi-onedimensional chains depending on their coverage ration.[11] Therefore, the adsorption not only depends on the nature of substrate but also on the adsorbed element and their coverage ratio.

The main motivation of the present work is to explore possible room temperature ferromagnetism in $\mathrm{MoS}_{2}$ through adsorption of light elements $(Y=\mathrm{H}, \mathrm{F}, \mathrm{Li})$ and strain using DFT and Stoner and Landau mean field models. The $Y$ atoms can be adsorbed on three possible sites, Mo-top, S-top, and hollow [28], among which the S-top site is the most stable [29]. The main effect of the adsorption of the $Y$-atoms on the monolayer is a shift of the Fermi energy into the $\mathrm{MoS}_{2}$ conduction or valence band, which dope the system and make it $n$-type or $p$-type, respectively, depending on the charge polarity of the $Y$ atom. We chose these elements due to their low formation energies and lack of clustering in $\mathrm{MoS}_{2}$ which usually occurs in transition metal dopants. These light elements are also important from an application point of view, such as for example green energy in the case of hydrogen. 


\section{METHOD OF COMPUTATION}

Density functional theory (DFT) [30] calculations, were performed with the plane-wave and pseudopotential method implemented in the Quantum Espresso package [31]. The exchange and correlation energy and potential were calculated with the Perdew-BurkeErnzerhof (PBE) parametrization [32] of the generalized gradient approximation (GGA). The ultrasoft pseudopotentials were parametrized with the recipe of Rappe, Rabe, Kaxiras and Joannopoulos [33]. For the spin-orbit coupling (SOC), the core electrons were treated fully relativistically. The electron wave function was expanded in a plane wave basis set, with a wave function- and charge-density cut-offs of $70 \mathrm{Ry}$ and $300 \mathrm{Ry}$, respectively. A $20 \times 20 \times 1$ Monckhorst-Pack grid [34] was used for the $k$-points, which gave a fine reciprocal-space

grid and hence a rather high accuracy. A vacuum slab of $15 \AA$ was used in the direction normal to the plane of $\mathrm{M} \mathrm{oS}_{2}$, to ensure the absence of interlayer interactions in that direction. The convergence of all computational parameters was checked carefully. Note for example that magnetism is very sensitive to k-point sampling. We have therefore carefully checked the convergence of magnetic moment, total energy, and electronic structure against all the computational parameters described above. In all these calculations we considered the most stable phase of $\mathrm{MoS}_{2}(2 \mathrm{H}$ trigonal prismatic $)$ and a single $Y$ atom adsorbed on the $\mathrm{S}$ site. We therefore focused on partially hydrogenated, Lithiated, and fluorinated $\mathrm{MoS}_{2}$, which led to uniform coverages of the $Y$ atoms without clustering (see Fig.1) similar to previous model used by Shi et al[8].

\section{RESULTS AND DISCUSSION}

The physical properties of a given material usually depend strongly on the lattice parameters. We first therefore calculate the total energy of $\mathrm{MoS}_{2}$ at different lattice constants, i.e. by applying an homogeneous strain, and fit the DFT data by using the BirchMurnaghan equation of state [35]. The calculated lattice constant of $2 \mathrm{D} \mathrm{MoS}_{2}$ is found to be $3.18 \AA$ (see Table SI) which is in agreement with previous calculations [36-38, 40]. Using the equilibrium lattice constant, the band structure of $2 \mathrm{D} \mathrm{MoS}_{2}$ is also calculated with and without SOC [see Fig. 2(a)]. The calculated direct bandgap at the $K$ point of $\mathrm{MoS}_{2}$ without SOC is $\sim 1.74 \mathrm{eV}$, which is also comparable to previous theoretical $[29,36-38]$ and experimen- 
tal values [39]. The direct bandgap behavior is different from bulk and a few-layers thick $\mathrm{MoS}_{2}$, which makes 2D MoS 2 a promising candidate material for electronic devices. We then used the GGA calculated lattice constant, and recalculated the band structure with SOC [(see Fig.2(a))]. In this last case the valence-band edge is splited due to the SOC, with the splitting being largest at the $K$-point of the Brillouin zone (BZ). Our GGA calculated SOC splitting is about $148 \mathrm{meV}$, which is not only consistent with previous theoretical calculations [40, 41], but also with the experimental value of $141 \mathrm{meV}$ [41]. Fig.2(a) also shows that the conduction band minimum, which also lies at the $K$-point, is doubly degenerated. The above calculations confirm the accuracy of our computational approach, since in general the correct SOC splitting is difficult to obtain and depends on the theoretical method [40].

For hydrogenated, lithiated, and fluorinated monolayer $\mathrm{MoS}_{2}$, the calculated total energies vs lattice constant give equilibrium lattice constants of $3.22,3.19$, and $3.21 \AA$ for H-, Li-, and F-doped $\mathrm{MoS}_{2}$, respectively. Noticeably, these added atoms do not generate too much lattice distortion in the host material. We also optimized the bond lengths of S-H, S-Li, and S-F (see Table SI) which turn out to be 1.41, 2.39, and $1.71 \AA$ respectively. Theses values are also comparable with previous DFT calculations [28, 29, 42-44]. The calculated formation energies are negative (see Table SI), which indicates that H, F, and Li can easily be adsorbed at $\mathrm{S}$ site in $\mathrm{MoS}_{2}$. The band structures of these systems were also calculated at the equilibrium geometry with and without spin-polarization. However, we found no signature of spin-polarization at such geometry, as opposed to the strained case, which will be discussed below. Note that that $\mathrm{t}$ he behavior of light elements, e.g. H, is different in systems such as graphene and silicene, where $\mathrm{H}$ not only induces magnetism without strain but also produces a rather large lattice distortion [45-47]. The calculated non spin-polarized band structures for H-, Li-, and F-doped $\mathrm{MoS}_{2}$ with and without SOC are shown in Fig.2(b-d). At fist glance it is clear that all the band structures show metallic behavior with and without SOC. Therefore, H, Li, and F induce metallicity in $\mathrm{MoS}_{2}$, and form impurity bands in the band-gap. When the calculations were repeated including the SOC effect, non-degenerate bands were also observed near the $K$-points in the BZ. The calculated SOC splitting is about 150, 130, and $150 \mathrm{meV}$ for $\mathrm{H}, \mathrm{Li}$, and $\mathrm{F}$ adsorbed $\mathrm{MoS}_{2}$, respectively. These values are comparable with the SOC splitting of pristine $\mathrm{MoS}_{2}$, although a slight decrease in this parameter can be seen in the lithiated case.

In agreement with previous calculations, which show that SOC is insensitive to pressure 
[48], we may conclude that the SOC in $\mathrm{MoS}_{2}$, is also insensitive to $\mathrm{H}, \mathrm{Li}$, and $\mathrm{F}$ added atoms. These atoms donate electrons to the host material and form electron bands just below the conduction band. In case of $\mathrm{H}$, the impurity band is not centred at the high symmetry points of the BZ. In contrast, Li forms a wide impurity band centred at the $G$ point of the $\mathrm{BZ}$, whereas the $\mathrm{F}$ impurity band is at the $K$ point. It is also interesting to note that all added atoms do not have bands at the $G$ point of the BZ and the conduction bands are well separated form the valance bands, except in the Li case, indicating strong metallicity in $\mathrm{MoS}_{2}$.

The above calculations were repeated using spin-polarized DFT, and no signature of spinpolarization was observed. We then strained the pristine material and calculated the band structures at different lattice constants. Again, no signature of magnetism was seen. In the strained $\mathrm{MoS}_{2}$ we found however a band gap transition from direct to indirect (not shown here), which is consistent with previous DFT calculations [23]. We then repeated the same calculations for the doped systems. First, for H-doped $\mathrm{MoS}_{2}$, we started from its equilibrium geometry and calculated the band structures at different lattice constants using both spinpolarized and non-spin-polarized calculations. Interestingly, we found metallic behavior at all lattice constants. The electronic density of states (DOS) at the Fermi energy $D\left(E_{\mathrm{F}}\right)$ of hydrogenated $\mathrm{MoS}_{2}$ at different lattice constants was also calculated to search for signatures of magnetism within the Stoner mean field mo del [49]. The calculated $D\left(E_{\mathrm{F}}\right)$ is shown in Fig.3(a) It is evident that $D\left(E_{\mathrm{F}}\right)$ for pristine $\mathrm{MoS}_{2}$ is almost zero as a function of the lattice constant until 3.6 $\AA$, where it becomes finite, indicating metallic behavior consistent with the notion of strain driven metallicity in $\mathrm{MoS}_{2}$ [23]. Hydrogenated $\mathrm{MoS}_{2}$, however, has always non-zero $D\left(E_{\mathrm{F}}\right)$, which increases with the lattice constant, until a rather large $D\left(E_{\mathrm{F}}\right)$ can be seen at around $3.4 \AA$. This large $D\left(E_{\mathrm{F}}\right)$ indicates possible magnetism in hydrogenated $\mathrm{MoS}_{2}$, according to Stoner's mean field model. Further increments of the lattice constant increase $D\left(E_{\mathrm{F}}\right)$ and the metallicity of hydrogenated $\mathrm{MoS}_{2}$.

We re-calculated $D\left(E_{\mathrm{F}}\right)$ including SOC, but no significant changes were observed. The SOC calculations also confirmed the onset of possible magnetism in hydrogenated $\mathrm{MoS}_{2}$. We then computed the spin-polarized $D\left(E_{\mathrm{F}}\right)$, i.e., $D\left(E_{\mathrm{F} \uparrow}\right)$ and $D\left(E_{\mathrm{F} \downarrow}\right)$, which are also shown in Fig.3(a). The spin-polarized $D\left(E_{\mathrm{F}}\right)$ remains degenerate as a function of the lattice constant until $3.40 \AA$ where it clearly splits into two opposite trends. The separation between $D\left(E_{\mathrm{F} \uparrow}\right)$ and $D\left(E_{\mathrm{F} \downarrow}\right)$ increases with the lattice constant indicating the presence of non-zero atomic 
magnetic moments.

Figure3(b) shows the spin magnetic moments per unit cell of hydrogenated $\mathrm{MoS}_{2}$. The magnetic moment is zero below $3.30 \AA$ consistent with the low $D\left(E_{\mathrm{F}}\right)$. However, above 3.30 $\AA$ which is about $2.5 \%$ strain, hydrogenated $\mathrm{MoS}_{2}$ becomes magnetic. Note that previous DFT calculations showed that $\mathrm{MoS}_{2}$ is thermodynamically stable under such a strain [50]. Further increments of the strain increase the magnetic moments until an strain of about $3.70 \%$, where the magnetic moments saturates at $1.0 \mu_{\mathrm{B}}$. Our detailed analysis shows that the increase in the magnetic moments with strain are mainly due to the $d$ electrons of Mo. The integral value of the magnetic moment indicates the half-metallic nature of hydrogenated $\mathrm{MoS}_{2}$, which will be discussed below. From these calculations, we can clearly conclude that it is easy to induce magnetism through strain in hydrogenated $\mathrm{MoS}_{2}$.

The spin-magnetic moments of lithiated and fluorinated $\mathrm{MoS}_{2}$ were also calculated [see Fig.3(b)]. Note, however, that large tensile strain $\sim 13 \%$ ( $12 \%$ ) are needed to induce magnetism in lithiated (fluorinated) $\mathrm{MoS}_{2}$. The observed magnetic moments in lithiated $\mathrm{MoS}_{2}$ are much smaller than those of hydrogenated and fluorinated $\mathrm{MoS}_{2}$ and no saturation of the magnetic moment can be seen in the studied range of lattice constants. The presence of small magnetic moments in lithiated $\mathrm{MoS}_{2}$ is consistent with experimental studies on nano tubes of Li-doped $\mathrm{MoS}_{2}$ [51]. Fluorinated $\mathrm{MoS}_{2}$ saturates to $1.0 \mu_{\mathrm{B}}$ at $\sim 18 \%$. We also calculated the magnetic moments of fluorinated $\mathrm{MoS}_{2}$ with SOC [see Fig.3(b)], but no significant change was observed, which confirms that the onset of magnetism and the saturation of the magnetic moment are insensitive to SOC.

To address the atomic origin of magnetism in all these systems and half-metallicity in $\mathrm{H} / \mathrm{F}$ added $\mathrm{MoS}_{2}$, we calculated the spin-polarized band structures at $3.70 \AA$ and $3.82 \AA$, which are shown in Fig.4(a, d). These are the lattice constants where the proposed materials show finite magnetic moments. At $3.70 \AA$ hydrogenated $\mathrm{MoS}_{2}$ has a fully spin-polarized band structure, where the spin-up component is metallic and the spin-down component insulating, giving rise to half-metallicity. Therefore, strained hydrogenated $\mathrm{MoS}_{2}$ can be regarded as a good candidate for spintronics devices. The spin-polarization is larger in the conduction band than in the valence band, which suggests that magnetism is mainly mediated by electrons. Isolated bands in the spin-up/spin-down states can also be seen just below the Fermi energy. Such bands are separated from each other through an exchange field of about $0.50 \mathrm{eV}$, indicating their relation to magnetism. Further strain $(3.82 \AA)$ only 
shifts the bands upwards and maintains almost the same exchange splitting.

The band structures of lithiated $\mathrm{MoS}_{2}$ at $3.70 \AA$ and $3.82 \AA$ are shown in Fig.4(b, e). In this case there is a negligible spin-polarization in the valence band. However, a small polarization near the Fermi energy is visible, which induces a magnetic moment of $0.15 \mu_{\mathrm{B}}$. The band structure remains metallic for both spin states, and even at $3.82 \AA$ has a metallic character. As expected, large strain induces larger magnetic moments, e.g. $0.36 \mu_{\mathrm{B}}$ at 3.82 $\AA$, but the valence band still remains roughly non-spin polarized. This means that the bands of lithiated $\mathrm{MoS}_{2}$ are very rigid against strain, as compared with $\mathrm{H}$ and $\mathrm{F}$ added $\mathrm{MoS}_{2}$. The origin of magnetism in lithiated $\mathrm{MoS}_{2}$ can also be explained with the Stoner mean field model by taking into account the increase of the DOS at the Fermi level.

For fluorinated $\mathrm{MoS}_{2}$, spin-polarized bands like those of the hydrogenated system can be clearly observed just below the Fermi energy at $3.70 \AA$ [see Fig.4(c, f)]. This system is metallic and has a magnetic moment of $0.36 \mu_{\mathrm{B}}$. However, at $3.82 \AA$ the spin splitting just below the Fermi energy increases and the system becomes half-metallic with a magnetic moment that saturates at $1.0 \mu_{\mathrm{B}}$. A large spin polarization can be seen in the conduction band as compared with the valence band, which again implies that magnetism is mediated by electrons. Half-metallic fluorinated $\mathrm{MoS}_{2}$ can therefore be another candidate material for spintronic devices.

To further elucidate the origin of strain-driven magnetism, we calculated the total and projected DOS of hydrogenated $\mathrm{MoS}_{2}$ [see Fig.5(a)]. At the equilibrium lattice constant this system has a band around $-2 \mathrm{eV}$ [see Fig.4(a)] which is mainly generated by Mo $d$ states, whereas the bands near the Fermi energy are related to Mo $d$ and S $p$ states. The metallicity is produced by the hybridization of Mo-S orbitals and also by the hydrogen states, which are present at the Fermi energy. Note that strained $\mathrm{MoS}_{2}$ is a semiconductor but here the $\mathrm{H}$ atoms drive metallicity through the Mo $d$ states. The spin-polarized DOS at $3.82 \AA$ Fig.5(a, d), shows that magnetism is mainly generated by the Mo- $d$ states. A rather large spin polarization around the Fermi energy can be seen at the Mo site for this lattice constant. The spin-up channel is metallic, whereas the spin-down DOS has a gap at the Fermi energy.

The projected DOS of lithiated $\mathrm{MoS}_{2}$ is also shown in Fig.5(b, e). In this case the metallicity is mainly dominated by the Li $s$ states, which generate a band that extends down to $1 \mathrm{eV}$ below the Fermi energy. The spin-polarized DOS at $3.82 \AA$ shows a small polarization near the Fermi energy but no polarization in the valence bands. This confirms the presence of 
electron mediated magnetism. The Mo $d$ orbitals give also a small contribution to magnetism, but the splitting is not very large as compared to the hydrogenated case. For the fluorinated system, the projected DOS is also shown in Fig.5(c, f). This case is very similar to the hydrogenated material: the system without strain is metallic, with bands around the Fermi level generated by Mo $d, \mathrm{~S} p$ and F $p$ states; the strained case is also half-metallic due to the Mo $d$ states.

The transition from the non-magnetic to the magnetic state in the strained $\mathrm{H},-\mathrm{Li}-$, and F-doped $\mathrm{MoS}_{2}$ can be describe with a Landau mean field model [49]. We carried out fixed moment calculations [52], calculated the total energy as a function of magnetic moment for different lattice constants (strain) and fitted the data with the Landau mean field model (see supplementary information). Figure 6 shows our calculated energy gain $\left(E(m)-E_{0}\right)$ as a function of the constrained spin magnetic moment $m$ under different lattice constants (tensile strains). The energy gain $\left(E(m)-E_{0}\right)$ can be defined as the difference between the total energies at zero and $m$ spin magnetic moments under the same strain. For hydrogenated $\mathrm{MoS}_{2}$ at a lattice constant of $3.40 \AA$ the energy gain has a global minimum at zero spin moment, which means that no-spontaneous magnetization is expected, in agreement with Fig.3(b). However, as the lattice const an $\mathrm{t}$ is increased, the energy gain achieves a global minimum at a finite spin moment $\left(\sim 1.0 \mu_{\mathrm{B}}\right)$, suggesting the onset of spontaneous magnetism. This implies that spontaneous half-metallic ferromagnetism is expected in strained hydrogenated $\mathrm{MoS}_{2}$. Similar results are also observed for Li-MoS 2 , where spontaneous magnetism appears at lattice constants $a \geq 3.60 \AA$ with a global minimum at $a=3.80 \AA$ and $\sim 0.4 \mu_{\mathrm{B}}$. Note again that $\mathrm{Li}-\mathrm{MoS}_{2}$ has a very small magnetic moment as compared with $\mathrm{H}$ - and F-added $\mathrm{MoS}_{2}$. This further confirms the experimentally observed small magnetization in Li-doped $\mathrm{MoS}_{2}$ nanotubes. A similar behavior can also be seen in fluorinated $\mathrm{MoS}_{2}$, where spontaneous magnetism appears at lattice constants $a \geq 3.60 \AA$. Fluorinated $\mathrm{MoS}_{2}$ has a global minimum at $a=3.80 \AA$ and $\sim 1.0 \mu_{\mathrm{B}}$. Therefore, spontaneous magnetism is also expected in strained F-doped $\mathrm{MoS}_{2}$. These observation s agree again with recent experimental work on F-added $\mathrm{MoS}_{2}$ [53], where magnetism is observed at room temperature.

Finally, to propose our simulated materials for practical applications, it is very important to investigate the ferromagnetic stability against antiferromagnetic structure of $Y$ doped $\mathrm{MoS}_{2}$. We considered larger supercells (e.g., $2 \times 2 \times 1$ ), included four $Y$ atoms at the top S sites and calculated the total energy of the supercell in the ferromagnetic and antiferromagnetic 
configurations. Our converged calculations reveal that the ferromagnetic configuration is more stable than the antiferromagnetic one for all $Y$ aded systems by $\sim 65 \mathrm{meV}$. Therefore, room temperature ferromagnetism is expected in our proposed systems. Note that the FM and AFM coupling was considered for strained $(3.82 \AA)$ systems. The zero strain system has no magnetism, so it is expected that the exchange interaction may increase with strain.

Before going to summarize our work, we would to like to comment on the clustering of $Y$ atoms. Note that we can not ignore the possibility of clustering of $Y(\mathrm{Li})$ atoms in $\mathrm{MoS}_{2}$ as observed in graphene[10]. We may speculate that clustering of Li atoms in $\mathrm{MoS}_{2}$ will be difficult as compared with clustering of $\mathrm{Li}$ in graphene[54]. Even such clustering of $Y$ atoms in $\mathrm{MoS}_{2}$, if present, can be degraded by the application of external electric field as shown in the case of graphene[10]. Further detailed investigations are required to consider all the possible clusters of $Y(\mathrm{Li})$ in $\mathrm{MoS}_{2}$ using the partial particle swarm optimization (PPSO) and DFT methods[10, 11].

\section{CONCLUSION}

Using density functional theory, we studied the electronic and magnetic properties of strained $Y$-doped $\mathrm{MoS}_{2}(Y=\mathrm{H}, \mathrm{Li}$, and $\mathrm{F})$. We found that pristine $\mathrm{MoS}_{2}$ is a direct band gap semiconductor, which becomes metallic when $\mathrm{H}, \mathrm{Li}$, and $\mathrm{F}$ are added at the $\mathrm{S}$ site. No sign of magnetism was observed at the equilibrium geometry with or without SO. We also

found that the DOS at the Fermi energy increases as a function of strain for the doped systems when external strain was imposed. The large DOS at the Fermi energy was used to propose the existence of strain-driven magnetism using Stoner's mean field model.

We calculated the spin magnetic moments as a function of strain and found that the most favourable case that induces magnetism is hydrogenated $\mathrm{MoS}_{2}$. The origin of magnetism was discussed in terms of charge transfer, based on the electronic structure. Half-metallic ferromagnetism was also predicted in $\mathrm{H}$ - and $\mathrm{F}$-doped $\mathrm{MoS}_{2}$. We used the Landau mean field theory to probe the emergence of spontaneous magnetism in the doped systems and found a good agreement between the fits and the ab-initio data. Finally, ferromagnetic and antiferromagnetic couplings were also studied and we found that the ferromagnetic state is more stable than the antiferromagnetic one. Based on these observations, we propose that 
H- and F- added $\mathrm{MoS}_{2}$ could be good candidate materials for spin-based electronic devices due to a $100 \%$ spin-polarization at the Fermi energy and ferromagnetism.

\section{ACKNOWLEDGMENTS}

GR acknowledges GIK Institute for providing supercomputing facility. V.M.G.-S. thanks the Spanish Ministerio de Economía, Industria y Competitividad for funding through the project FIS2015-63918-R. 
[1] G. Rahman, N. Ud Din,V. M. García-Suárez, and E. Kan, Phys. Rev. B 87, 205205 (2013).

[2] H. Ohno, H. Munekata, T. Penney, S. von Molnar, and L. L. Chang, Phys. Rev. Lett. 68, 2664 (1992).

[3] G. Rahman ,V. M. García-Suárez, and J. M. Morbec, J. Mag. Mag. Mater. 328, 104 (2013)

[4] A. K. Geim, Science 324, 1530 (2009).

[5] K. S. Novoselov, D. Jiang, F. Schedin, T. J. Booth, V. V. Khotkevich, S. V. Morozov, and A. K. Geim, Proc. Natl. Acad. Sci. U.S.A. 102, 10451 (2005).

[6] G. Rahman, Euro Phys. Lett. 105, 37012 (2014).

[7] A. Azcatl, X. Qin, A. Prakash, C. Zhang, L. Cheng, Q. Wang, N. Lu, M. J. Kim, J. Kim, K. Cho, R. Addou, C. L. Hinkle, J. Appenzeller, and R. M. Wallace, Nano Lett. 16, 5437 (2016).

[8] H. Shi, H. Pan, Y. W. Zhang, and B. I. Yakobson, Phys. Rev. B 88, 205305 (2013).

[9] J.Zhou,Q. Sun, Q. Wang,and P. Jena,Phys. Rev. B 90, 205427 (2014).

[10] J.Zhou,Q. Sun, Q. Wang,and P. Jena,Phys. Rev. B 90, 064505 (2015).

[11] J. Zhou, S. Zhang, Q. Wang, Y. Kawazoe, and P. Jena, Nanoscale 7, 2352 (2015).

[12] Y. Li, P. Chen, G. Zhou, J. Li, J. Wu, B. Gu, S. B. Zhang, and W. Duan, Phys. Rev. Lett. 109, $206802(2012)$.

[13] H. Shi, H. Pan, Y. W. Zhang, and B. I. Yakobson, Phys. Rev. B 87, 155304 (2013).

[14] K. F Mak,K. L. McGill,J Park, and P. L. McEuen, Science 344, 1489 (2014).

[15] Lberto F. Morpurgo, Nature Physics 9, 532 (2013).

[16] X. Xu, W. Yao, D. Xiao and T. F. Heinz, Nature Physics 10, 343 (2014).

[17] W. K. Ho, J. C. Yu, J. Lin, J. G. Yu, and P. S. Li, Langmuir 20, 5865 (2004).

[18] B. Radisavljevic, A. Radenovic, J. Brivio, V. Giacometti, and A. Kis, Nature. Nanotecnology. 6, 147 (2011).

[19] A. N. Enyashin, L. Yadgarov, L. Houben, I. Popov, M. Weidenbach, R. Tenne, M. B. Sadan, and G. Seifert, J. Phys. Chem. C, 115, 24586 (2011).

[20] I. Natalia , O. D. Denis, A. Vitaliy ,O. Umit, M. Hadis, Turk. J. Phys. 38, 478 (2014).

[21] H. Wang, H. Yuan, S. S. Hong, Y. Li and Y. Cui, Chem. Soc. Rev 44, 2664 (2015).

[22] S. Tongay,J. Zhou,C. Ataca,K. Lo, T. S. Matthews,J. Li,J. C. Grossman, J. Wu, Nano Lett., 12, 5576 (2012). 
[23] W. S. Yun, S. W. Han, S. C. Hong, I. G. Kim, and J. D. Lee, Phys. Rev. B 85, 033305 (2012).

[24] X. Duan, C. Wang, Z. Fan, G. Hao, L. Kou, U. Halim, H. Li, X. Wu, Y. Wang, J. Jiang, A. Pan, Y. Huang, R. Yu, and X. Duan, Nano Lett., 16, 264 (2016).

[25] C. Ataca, S. Ciraci, Journal of Phys. Chem. C 11513303 (2011).

[26] F. Zheng, Z. Liu, J. Wu, W.Duan, and B. L. Gu, Phys. Rev. B 78, 085423 (2008).

[27] J. D. Fuhr and A. Saul, Phys. Rev. Lett. 92, 026802 (2004).

[28] K. Dolui, I. Rungger, C. D. Pemmaraju, and S. Sanvito, Phys. Rev. B 88 075420(2013).

[29] E. W. K. Koh, C. H. Chiu, Y. K. Lim, Y-W. Zhang, H. Pan, International journal of hydrogen energy 3714323 (2012).

[30] Hohenberg P, Kohn W. Phys. Rev. B 136, 71 (1964).

[31] Giannozzi, S. Baroni, N. Bonini, M. Calandra, R. Car, C. Cavazzoni, D. Ceresoli, D. L. Chiarotti, M. Cococcioni, I. Dabo, A. D. Corso, S. de Dironcoli, S. Fabris, G. Fratesi, R. Gebauer, U. Gerstmann, C. Gougoussis, A. Kokalj, M. Lazzeri, L. Martin-Samos, N. Marzari, F. Mauri, R. Mazzarello, S. Paolini, A. Pasquarello, L. Paulatto, C. Sbraccia, S. Scandolo, G. Sclauzero, A. P. Seitsonen, A. Smogunov, P. Umari, R. M. Wentzcovitch, J. Phys. Condens. Matter 21, 39 (2009).

[32] J. P. Perdew, K. Burke, and M. Ernzerhof, Phys. Rev. Lett. 77, 3865 (1996).

[33] A. M. Rappe, K. M. Rabe, E. Kaxiras, and J. D. Joannopoulos, Phys. Rev. B 41, 1227(R)(1990); Erratum Phys. Rev. B 4413175 (1991).

[34] Monkhorst, H.J. and Pack, J.D. Phys. Rev. B 13, 5192 (1976).

[35] F. Birch, Phys. Rev. Lett. 71, 809 (1947). F. D. Murnaghan, Proc. Natl Acad. Sci. USA 30, 244 (1944).

[36] P. Johari, V. B. Shenoy, ACS, Nano 65449 (2012)

[37] S.M. Tabatabaei, M. Noei, K. Khaliji, M. Pourfath and M. Fathipour, J. Appl. Phys. 113, 163708 (2013).

[38] E. Scalise, M. Houssa, G. Pourtois, V. Afanasev, and A. Stesmans, Nano Res. 543 (2012).

[39] K. F. Mak, C. Lee, J. Hone, J. Shan, and T. F. Heinz, Phys. Rev. Lett. 105, 136805 (2010).

[40] A. Ramasubramaniam, Phys. Rev. B, 86, 115409 (2012).

[41] D. Xiao, G.B Liu, W. Feng, X. Xu and W. Yao. Phys. Rev. Lett. 108, 196802 (2012)

[42] J. He, K. Wu, S. Rongjian, L. Qiaohong, and W. Yongqin, App. Phys. Let. 96, 082504 (2010).

[43] X. Sun, Z. Wang and Y. Q. Fu, Scientific Reports 5, 18712 (2015). 
[44] F. Ersan, G. Gokoglu and E. Aktiirk, J. Phys. Chem. C 11928648 (2015).

[45] B. S. Pujari, S. Gusarov, M. Brett, and A. Kovalenko1, Phys. Rev. B 84, 041402 (2011).

[46] J. Zhou, Q. Wang, Q. Sun, X. S. Chen,Y. Kawazoe, and P. Jena, Nano Latt. 9, 3867 (2009).

[47] M. Houssa, E. Scalise, K. Sankaran,G. Pourtois, V. V. Afanasev, Appl. Phys. Lett. 98, 223107 (2011)

[48] X. Dou, K. Ding, D. Jiang, X. Fan, and B. Sun, ASC Nano, 10, 1619 (2016).

[49] S.Blundell, Magnetism in Condensed Matter (Oxford Master Series in Condensed Matter Physics) 2001.

[50] H.Soni and P. K. Jha, AIP Advances, 5, 107103 (2015).

[51] D. Mihailovic, Z. Jaglicic, D. Arcon, A. Mrzel, A. Zorko, M. Remskar, V.V. Kabanov, R. Dominko, M. Gaberscek, C. J. Gómez-García, J.M. Martínez-Agudo, and E. Coronado Phys. Rev. Lett. 90, 146401 (2003).

[52] J. Häglund, Phys. Rev. B 47, 566 (1993).

[53] D. Gao, S. Shi, K. Tao, B. Xiaa, and D. Xue, Nanoscale, 7, 4211 (2015).

[54] We carried out some additional test calculations on the clustering of $\mathrm{Li}$ in $\mathrm{MoS}_{2}$ by considering a triangular cluster and a square cluster (uniform distribution), our preliminary binding energy per $\mathrm{Li}$ results show that the uniform distribution of $\mathrm{Li}$ is more stable than the triangular distribution of $\mathrm{Li}$ in $\mathrm{MoS}_{2}$. 


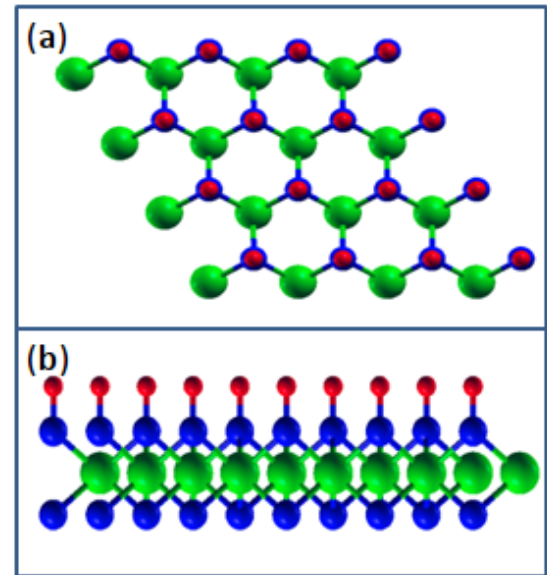

FIG. 1. (Color online) I will add. 
(a)

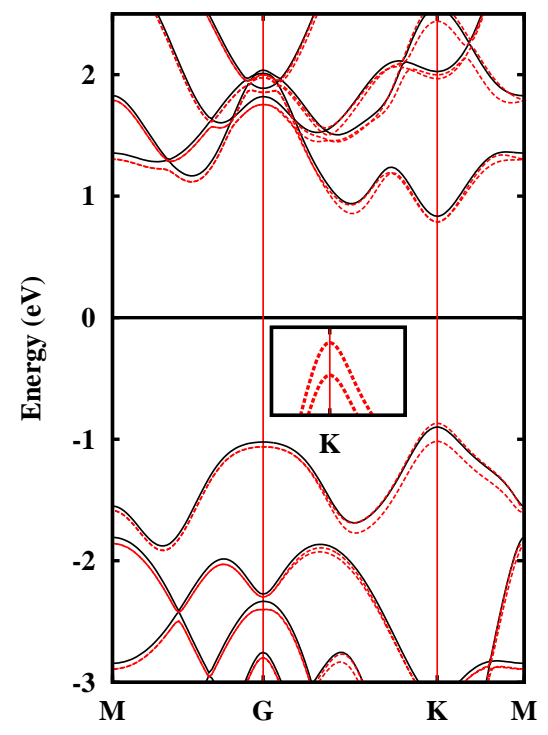

(c)

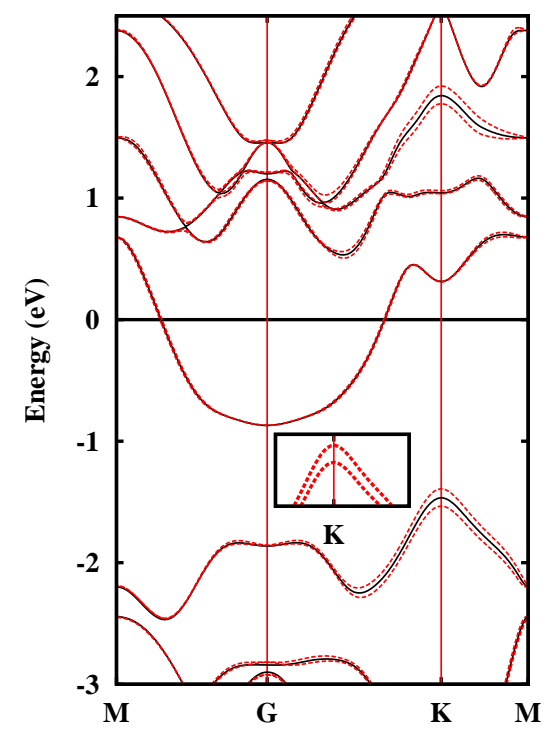

(b)

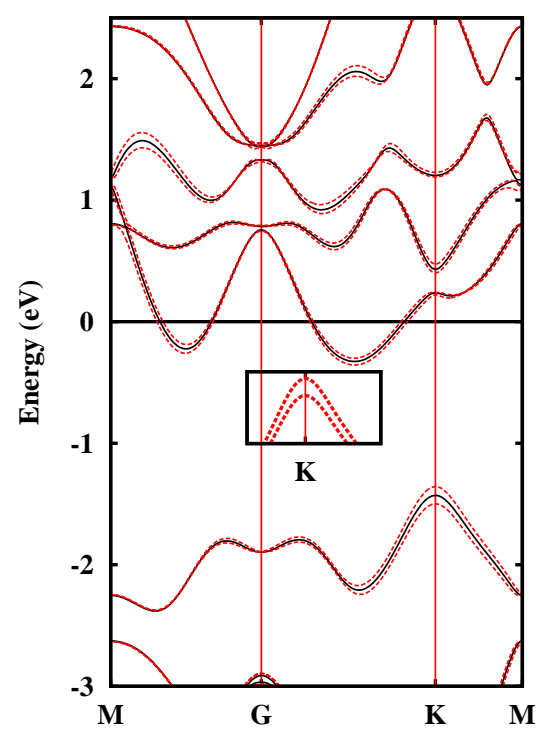

(d)

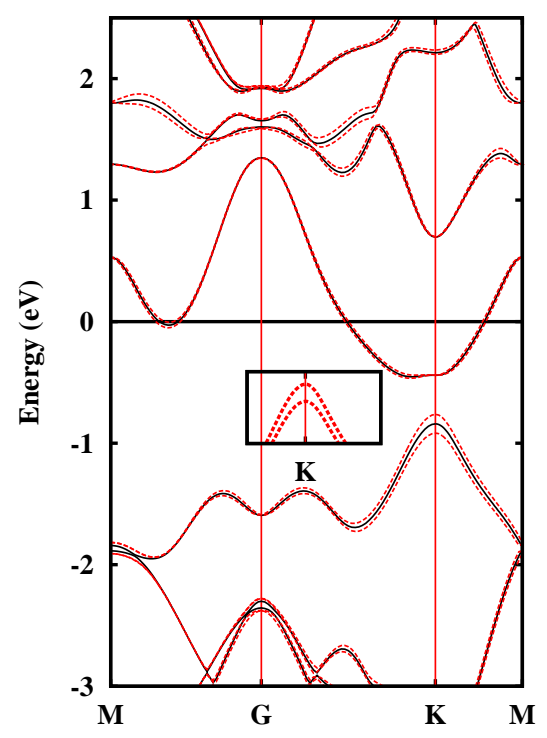

FIG. 2. (Color online) Calculated band structures of pristine $\mathrm{MoS}_{2}$ (a), hydrogenated $\mathrm{MoS}_{2}$ (b), lithiated $\mathrm{MoS}_{2}$ (c), and fluorinated $\mathrm{MoS}_{2}$ (d) calculated at their equilibrium lattice constants without SOC (solid lines) and with SOC (dashed lines). The inset shows the SOC coupling at the $K$-point of the BZ. 

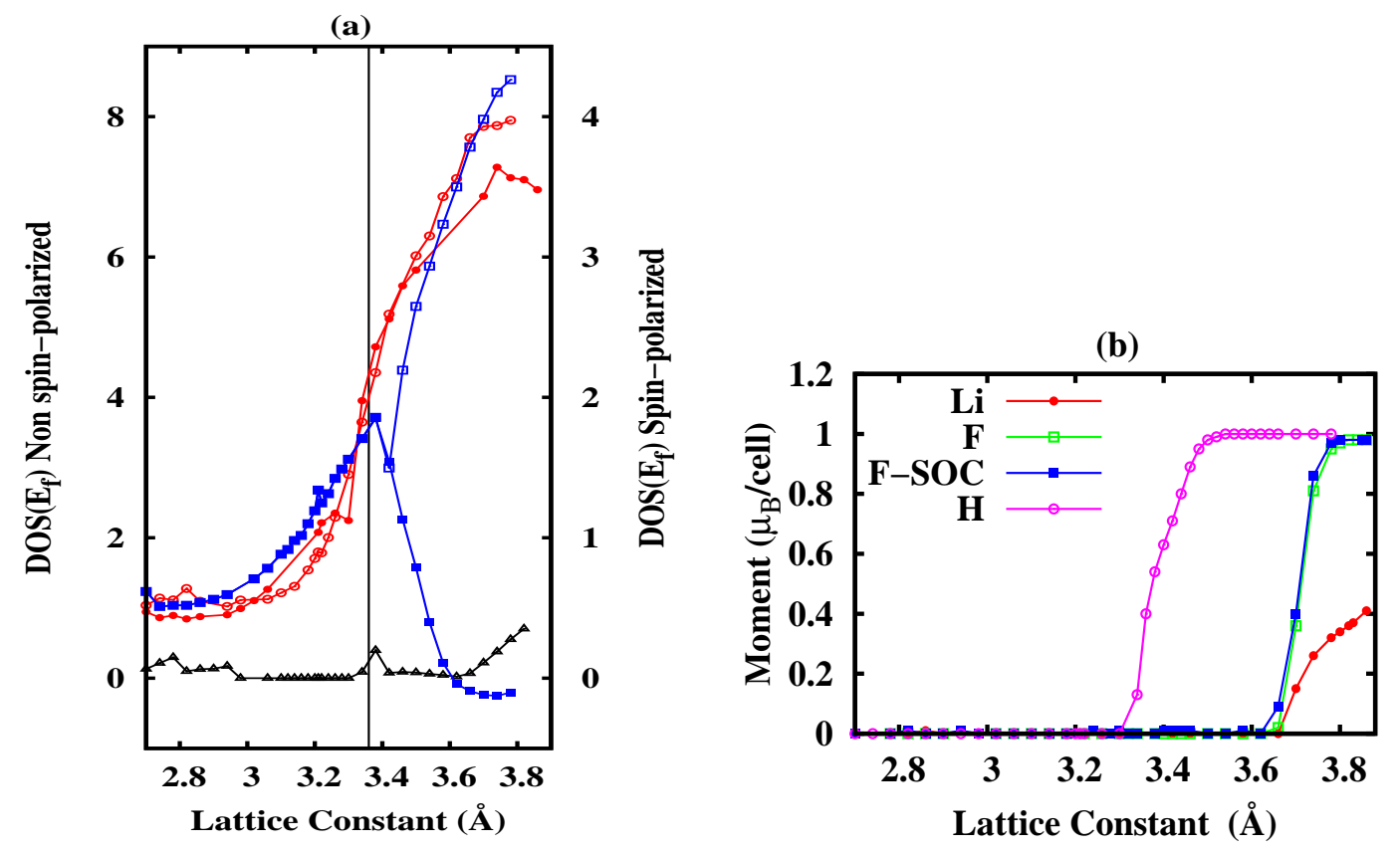

FIG. 3. (Color online) Density of states of pristine $\mathrm{MoS}_{2}$ and hydrogenated $\mathrm{MoS}_{2}$ calculated at the Fermi energy, $g\left(E_{\mathrm{F}}\right)$, vs lattice constant. Triangles represent $g\left(E_{\mathrm{F}}\right)$ of pristine $\mathrm{MoS}_{2}$, solid circles $g\left(E_{\mathrm{F}}\right)$ of hydrogenated $\mathrm{MoS}_{2}$ without SOC, empty circles $g\left(E_{\mathrm{F}}\right)$ with SOC, and filled (empty) squares spin-polarized $g\left(E_{\mathrm{F}}\right)$ for spin up (spin-down) states. The vertical line indicates the lattice constant at which the magnetization starts to develop. 
(a)

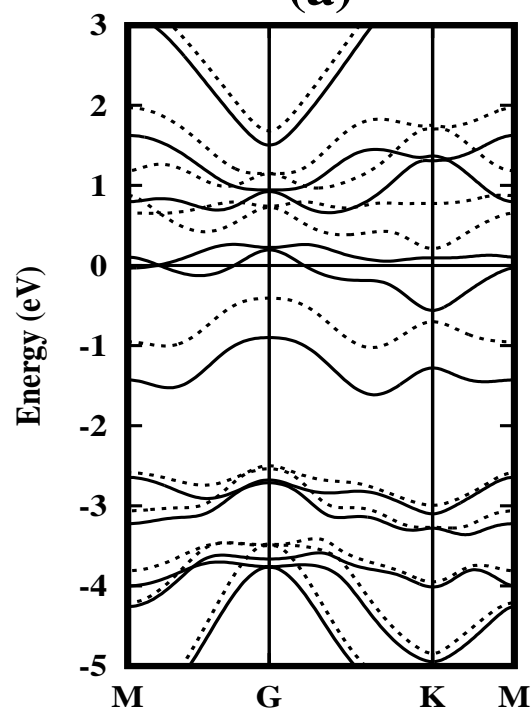

(d)

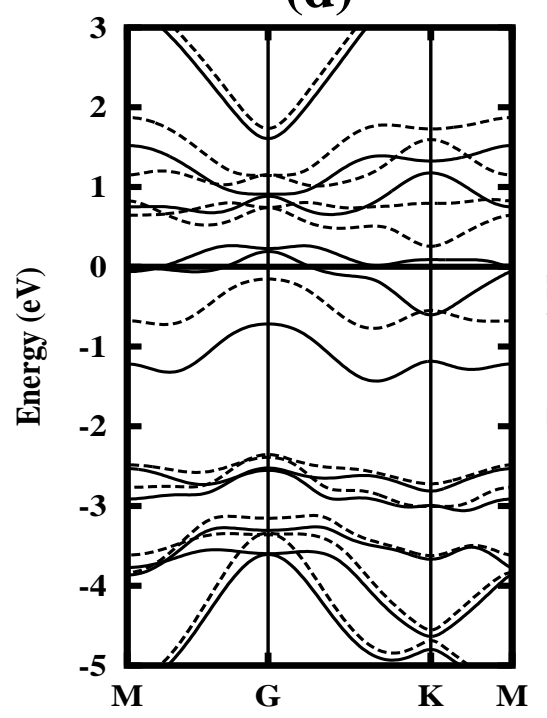

(b)

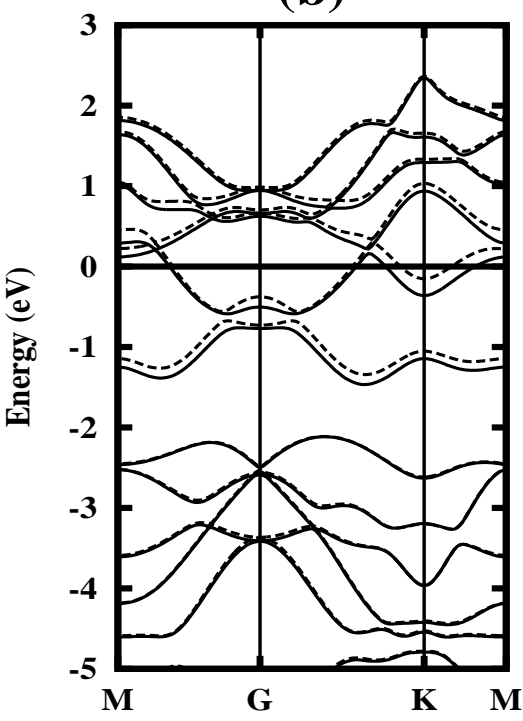

(e)

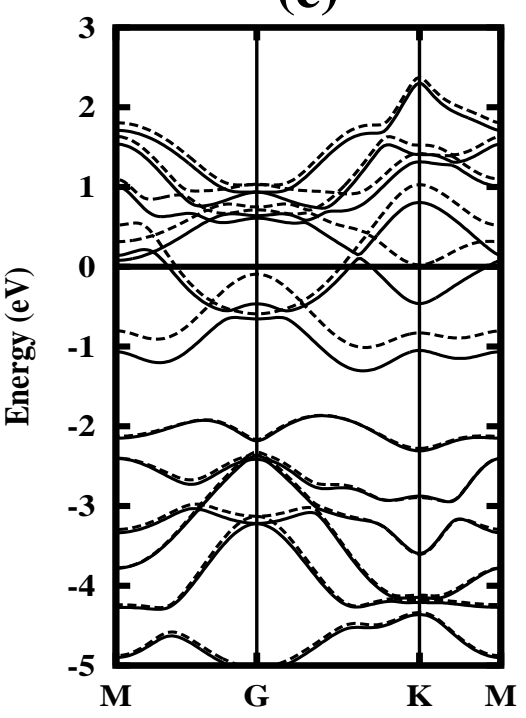

(c)

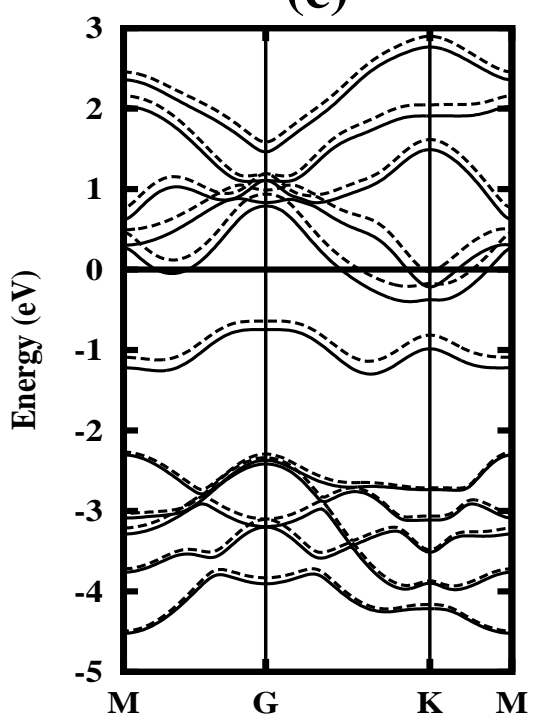

(f)

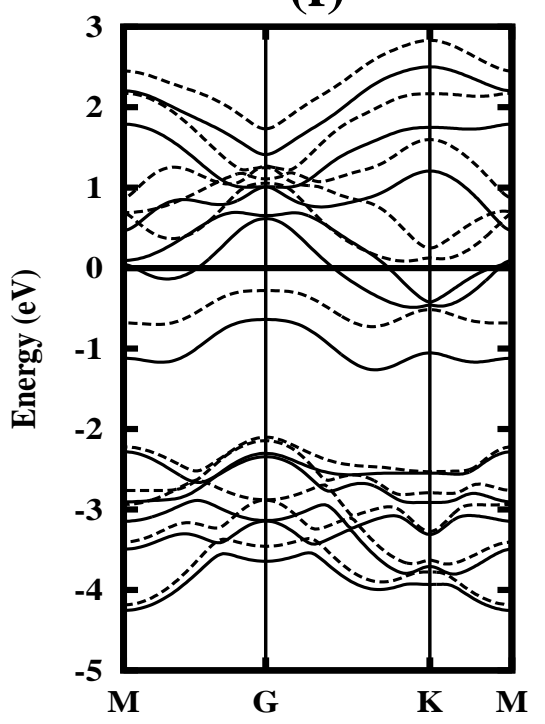

FIG. 4. (Color online) Spin-polarized band structure of hydrogenated $\mathrm{MoS}_{2}$ calculated at lattice constants of (a) $3.70 \AA$, and (d) $3.82 \AA$, spin-polarized band structure of lithiated $\mathrm{MoS}_{2}$ calculated at lattice constants of (b) $3.70 \AA$, and (e) $3.82 \AA$ and spin-polarized band structure of fluorinated $\mathrm{MoS}_{2}$ calculated at lattice constants of (c) $3.70 \AA$, and (f) $3.82 \AA$. Solid (dotted) lines represent spin-up (spin-down). The horizontal line indicates the Fermi energy, which is set to zero. 
(a)

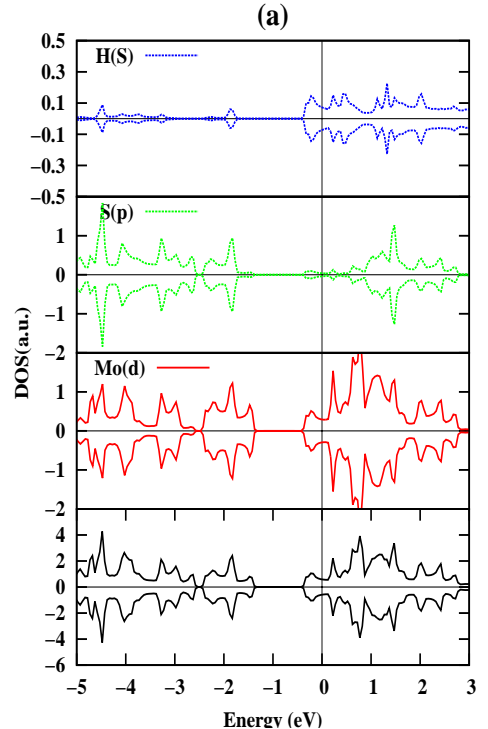

(d)

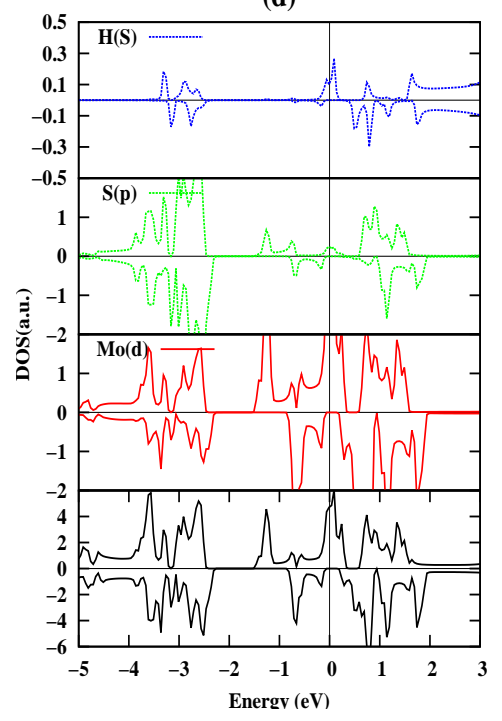

(b)

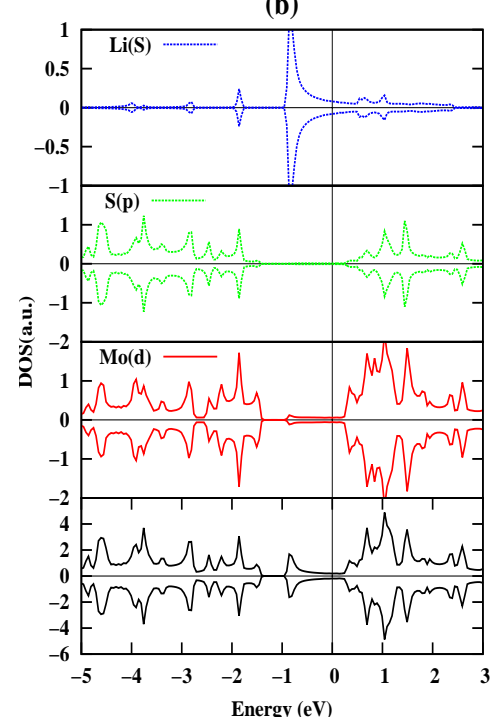

(e)

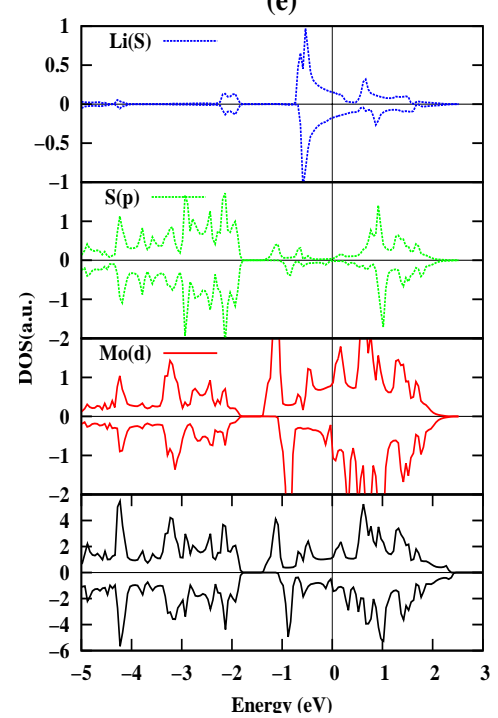

(c)

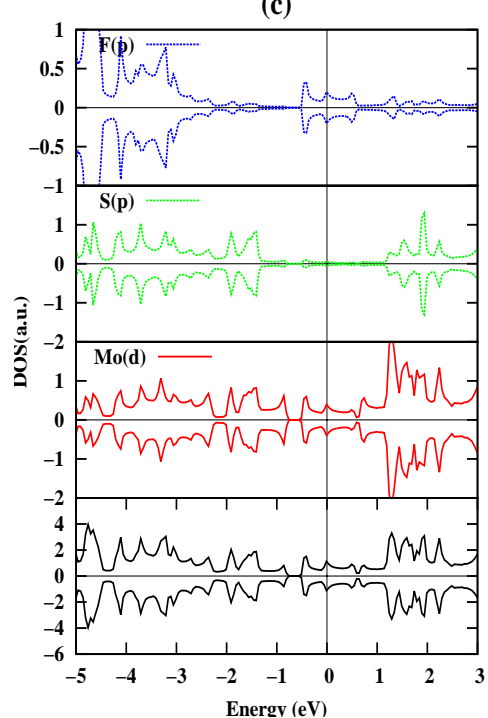

(f)

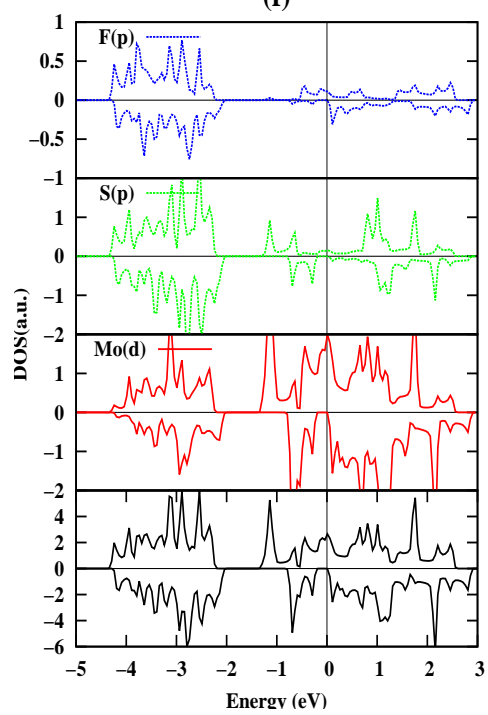

FIG. 5. (Color online) Total and partial density of states (PDOS) of hydrogenated $\mathrm{MoS}_{2}$ calculated at (a) the equilibrium lattice constant and (d) $3.82 \AA$ lithiated $\mathrm{MoS}_{2}$ calculated at (b) the equilibrium lattice constant and (e) $3.82 \AA$ and fluorinated $\mathrm{MoS}_{2}$ calculated at (c) the equilibrium lattice constant and (f) $3.82 \AA$. Black (solid), red (solid), green (dashed), and blue (dotted) lines represent total DOS and PDOS of $\mathrm{Mo}(d), \mathrm{S}(p)$, and $\mathrm{H} / \mathrm{Li} / \mathrm{F}(s)$ atoms, respectively. The Fermi energy is set to zero. The upper (lower) panels shows spin-up (spin-down). 
(a)

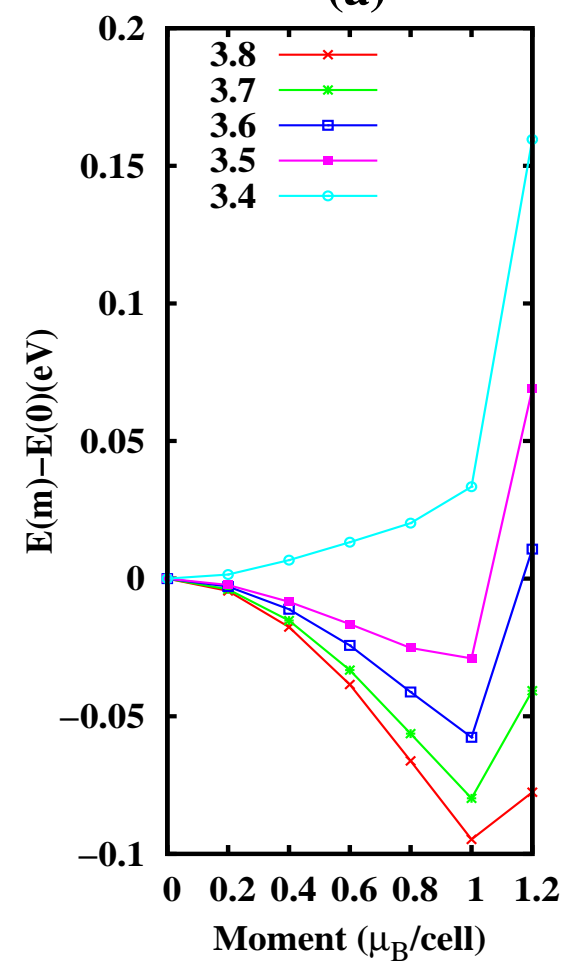

(b)

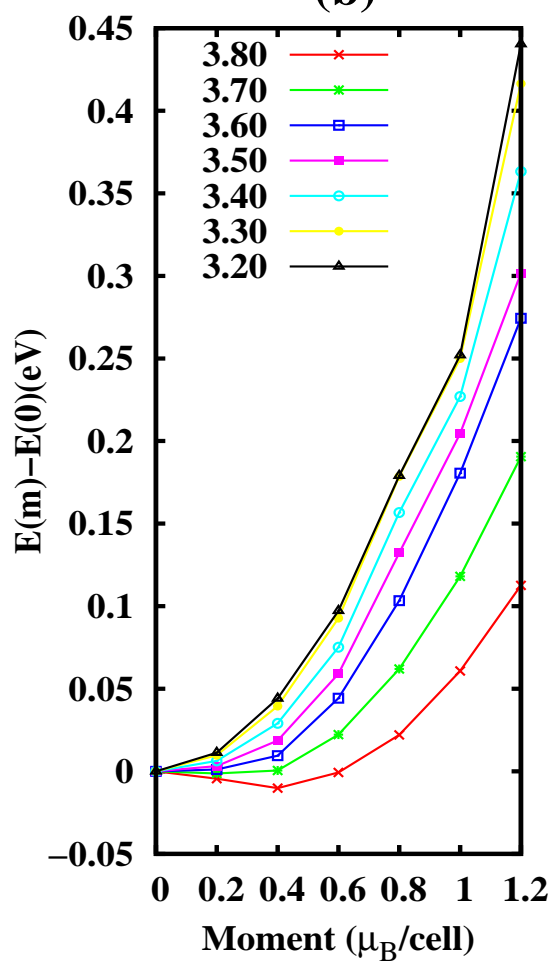

(c)

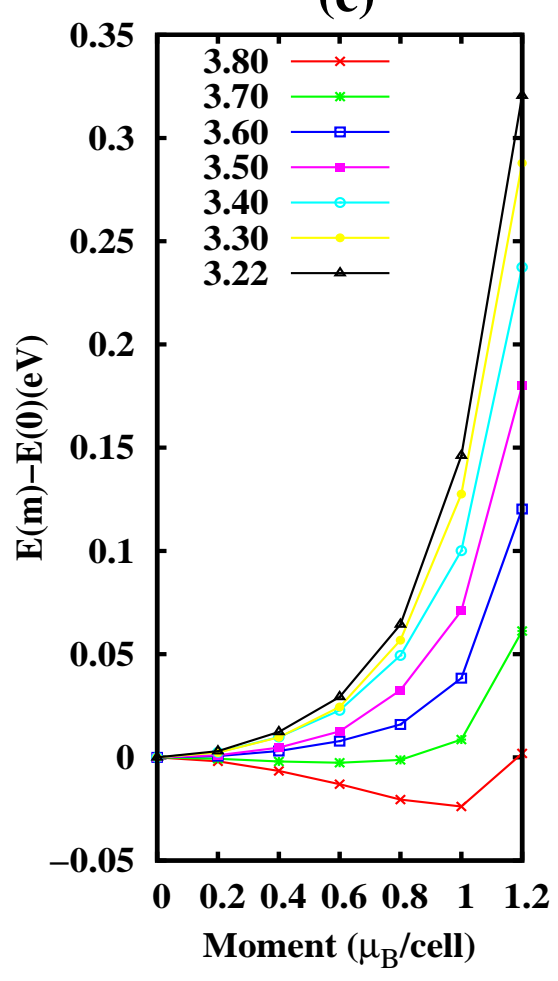

FIG. 6. (Color online) Relative energies $(\mathrm{eV})$ versus magnetic moment per unit cell $\left(\mu_{B}\right)$ for hydrogenated (a), lithiated (b) and fluorinated (c) $\mathrm{MoS}_{2}$ calculated at different lattice constants $(\AA)$. 